\section{Giant virilizing adrenocortical carcinoma in a girl presenting with mutism}

\author{
Huseyin Demirbilek, ${ }^{1}$ Serhan Kupeli,2 \\ Mehmet Nuri Ozbek,2 Ahmet Baran, 3 \\ Cigdem Murat Kemal,4 Ozekinci Selver ${ }^{4}$ \\ 1Department of Pediatric Endocrinology, \\ Diyarbakir Children Diseases Hospital, \\ Diyarbakir; 2Department of Pediatric \\ Oncology, Diyarbakir Children Diseases \\ Hospital, Diyarbakir; ${ }^{3}$ Department of \\ Radiology, Diyarbakir Children Diseases \\ Hospital, Diyarbakir; ${ }^{4}$ Department \\ of Pediatric Surgery, Dicle University, \\ Diyarbakir; ${ }^{5}$ Department of Pathology, \\ Dicle University, Diyarbakir, Turkey
}

\section{Abstract}

Adrenocortical tumors (ACT) are rare tumors of childhood. There are some difficulties in classification of pediatric ACT as adenoma or carcinoma. Prognostic characteristics as well as treatment choices are controversial. In general virilizing ACT are presenting at earlier age than non-functional tumors. Here we presented an early presented, delayed diagnosed giant virilizing adrenocortical tumor in a girl presented with mutism. Present case is interesting both in complaints at the presentation and the nature of tumor biology. Based on previously reported prognostic criteria present case had borderline characteristics and treatment option was only total resection of the tumor without adjuvant chemotherapy.

\section{Introduction}

Pediatric adrenocortical tumors (ACT) are rare and account only $0.2 \%$ of childhood tumors. ${ }^{1}$ Adrenocortical carcinoma (ACC) is an extremely unusual, and highly malignant childhood tumor. Its incidence is reported 0.3 cases per million per year in children. ${ }^{2}$ Bimodal age distribution occurs with the first peak occurring before five years of age and the second peak in the fourth to fifth decade of life. Adrenal tumors can be classified as functional (FT) when their hormonal secretions result in clinical consequences: Cushing syndrome, virilization syndrome, feminization syndrome, or a mixed Cushing-virilizing syndrome. Tumors are considered non-functional (NF) when the tumors do not secrete excessive hormones or produce hormonal precursors and/or active hormones in quantities insufficient to have clinical consequences. ${ }^{3}$ In children patients with functional ACT generally present with virilization signs or stigmata of Cushing syndrome..$^{3-5}$ In clinical practice signs and symptoms of secreted hormones provide diagnosis of functional adrenocortical tumors at early age comparing to non-functional tumors. We describe a giant virilizing ACC in a girl presented with complaint of mutism caused by deepening in her voice.

\section{Case Report}

A 5.5 year-old girl was admitted to our clinic with mutism caused by deepening in her voice. Previous history revealed that she was born as fourth child of non-consanguinous parents, after a 39 weeks pregnancy and uneventfull delivery. Her birthweight was 3000 gr. She was admitted to another hospital at the age of 1 year with the complaint of apperarence of pubic and axillary hair. She has been evaluated for congenital adrenal hyperplasia and none of imaging studies was performed. Clinical follow up was recommended. However, the parents could not have brought the child to the regular follow up because of social\&economic deprivation. During subsequent years, clinical progression of virilization have resulted in deepening of voice and ultimately a voluntary mutism have occured which was the chief complaint for admission to our clinic.

At the time of admission, in physical examination her weight was $28 \mathrm{~kg}$ (97 percentile), height was $120.7 \mathrm{~cm}$ (90-97 percentie). There were signs of virilization (deepening in voice, hyperpigmentation in genital area and areola, facial acnea, hirsutismus and clitoromegaly). Tanner staging of pubertal development was revealed stage 1 breast development, presence of axillary hair and stage 4 pubic hair. Laboratory examination showed elevated adrenal androgens (Table 1). Radiological investigations revealed advanced bone age (10.5 years). Abdominal ultrasonography and computerized tomography showed a large $(13 \times 11.4$ $\mathrm{cm})$ cystic, heterogeneous mass on right adrenal localization (Figure 1). There was no sign of metastases in PET scan.

Clinical and laboratory findings were consistent with virilizing adrenocortical tumor and the patient underwent surgical resection.
Correspondence: Huseyin Demirbilek, Pediatric Endocrinologist, Diyarbakir Children Diseases Hospital, Pediatric Endocrinology Unit, Diyarbakir, Turkey.

Tel. +90.412.224 5751 - Fax. +090.412.2240183

E-mail: dr_huseyin@hotmail.com

Key words: virilizing adrenocortical carcinoma, premature adrenarche, Weiss criteria.

Conflict of interest: the authors report no conflicts of interest.

Received for publication: 26 May 2011.

Revision received: 12 July 2011.

Accepted for publication: 14 July 2011.

This work is licensed under a Creative Commons Attribution NonCommercial 3.0 License (CC BYNC 3.0)

(C) Copyright H. Demirbilek et al., 2011

Licensee PAGEPress, Italy

Endocrinology Studies 2011; 1:e9

doi:10.4081/es.2011.e9

Complete resection of tumoral mass with negative surgical margin was succesfully performed without perioperative complication. Resected tumor was $14 \times 12 \times 5 \mathrm{~cm}$ in diameter and $750 \mathrm{gr}$ in weight, with regular border, containing solid and cystic components, central necrosis and grayish, relatively fragile specimen. Histopa thological examination revealed that there was a fibrous capsule which seperated tumor from peripheral tissues. There was not capsular invasion. Tumoral cells were pleomorphic, had

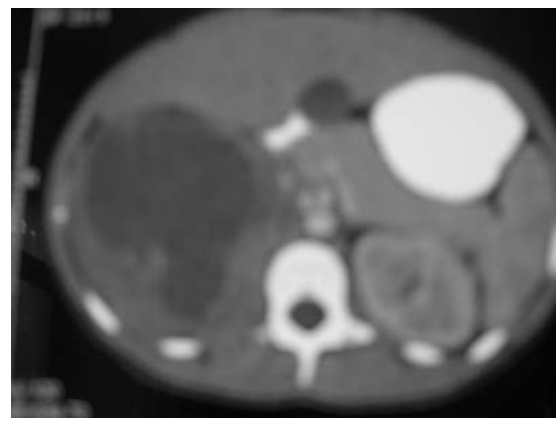

Figure 1. Computerized tomography image of tumoral mass on the right adrenal localization.

Table 1. Hormonal evaluation before and after surgical resection of tumor

\begin{tabular}{lccc} 
Hormone & Pre-operative & Pos-toperative & Normal range \\
Testosteron $(\mathrm{ng} / \mathrm{dL})$ & 629 & $<20$ & $<20$ \\
DHEAS $(\mu / \mathrm{dL})$ & 1543 & $<15$ & $0-60$ \\
\hline $17(\mathrm{OH})$ progesteron $(\mathrm{ng} / \mathrm{mL})$ & 15.5 & 0.3 & $0-1,8$ \\
Cortisol $(\mu / \mathrm{mL})$ & 35.9 & 1.72 & $5-15$ \\
\hline ACTH $(\mathrm{pg} / \mathrm{mL})$ & 9.22 & 75.8 & $0-46$ \\
\hline
\end{tabular}


wide eosinophilic-vacuolizated cytoplasm and large hyperchromatic nucleus (Figure $2 \mathrm{a}, \mathrm{b}$ ). Immunohystochemical examination showed positive staining with synaptophysin, negative staining with pan-cytokeratin (PCK), chromogranin, melan A, HMB45 and vimentin (Figure 2 c). Ki-67 proliferation index was $25 \%$ (Figure $2 \mathrm{~d})$. The present case consisted of 5 of 9 Weiss criteria. 6,7 This criteria was high nuclear grade, mitotic rate six or more per 50 high power fields, atypical mitoses diffuse architecture pattern more than $1 / 3$ of the tumor and confluent necrosis. Both with clinical and histopathologic findings according to Weiss criteria a diagnosis of carcinoma was concluded rather than
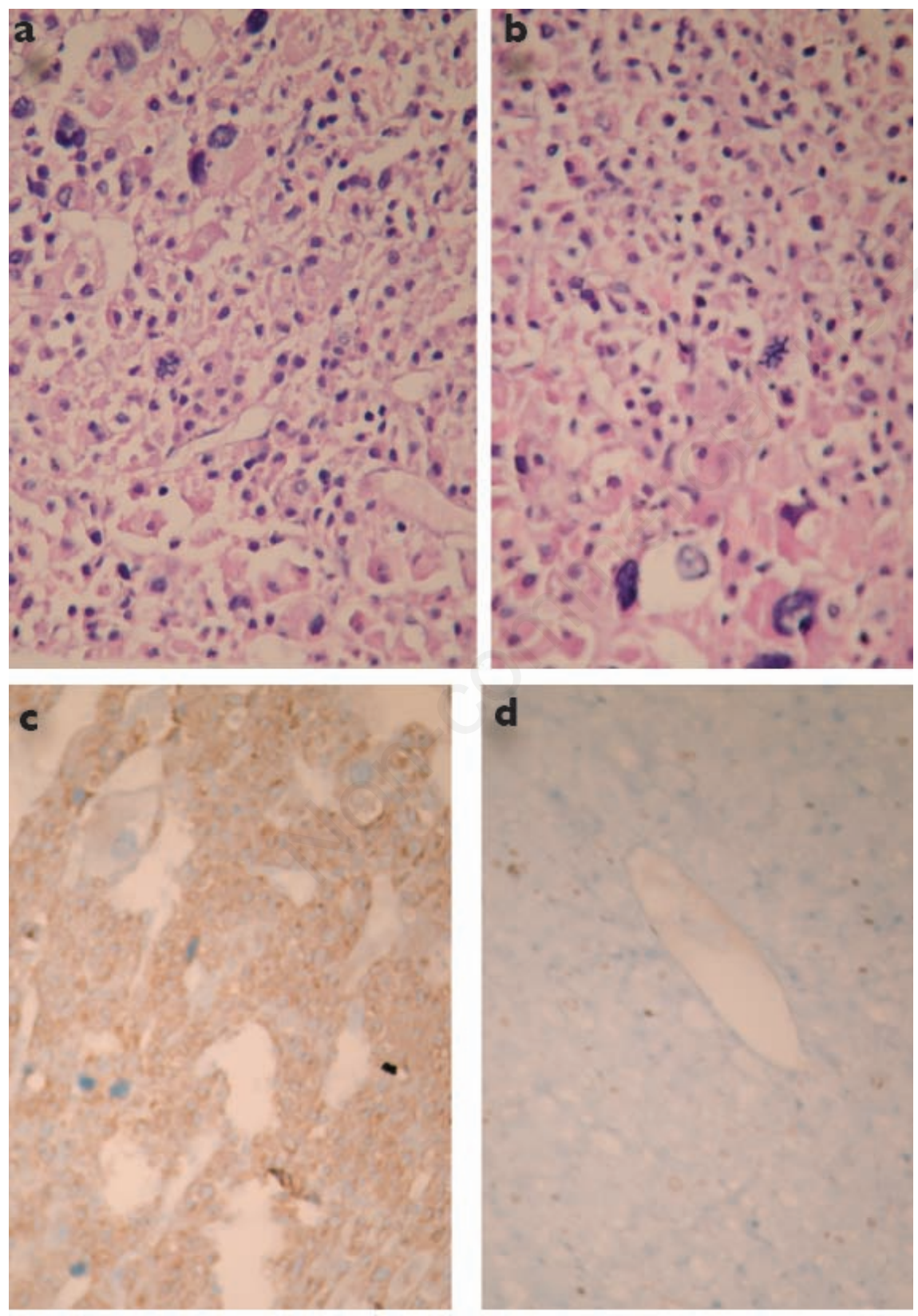

Figure 2. (a, b) Multipolar atypic mitoses in pleomorphic cells with eosinophilic cytoplasm and high nuclear grade (400X, H\&E staining); (c) Synaptophysin (+) immuneperoxidase staining cells ( 200X), (d) immuneperoxidase, Ki-67 (400X).

adenoma. Perioperative and post operative steroid replacement therapy was given to prevent adrenal insufficieny. After operation complete hormonal resolution was observed (Table 1). Patient is under close follow up and in complete remission without adjuvant chemotherapy at fifth month after surgery.

\section{Discussion}

Adrenocortical carcinoma of childhood is a rare but highly malignant tumor. Functional ACT present with signs and symptoms of viril-

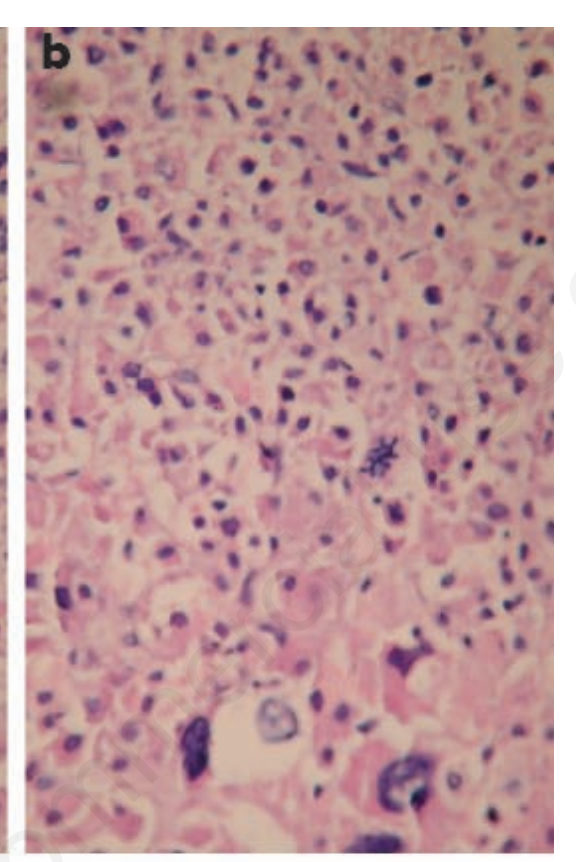

Regarding staging in our case, the tumor was resected with negative surgical margins and there was no evidence of metastases. However, tumor size was too large $(750 \mathrm{~g})$. Complete hormonal resolution was achieved after surgery. Thus, present case was stage II, low grade adrenocortical carcinoma. ${ }^{11}$

Since pediatric ACT are extremely rare and clinical expression is heterogenous, prognostic factors have been difficult to establish in this age group.5,11 Evaluation of prognostic criteria in our case revealed that large tumoral mass (750 gr), high mitotic index, high Ki-67 proliferation index (25\%), atypical mitoses, age at the time of the diagnosis and long duration between the initial symptoms and the diagnosis were poor prognostic criteria.11 However, total resection of the tumor with negative surgical margins, absence of vascular and capsular invasion, confluent necrosis, absence of metastasis, complete resolution of the hormonal and the clinical findings were in favor of good prognosis.5,6,11 It is accepted that, surgery is the single most important procedure in the successful treatment of ACT. The role of chemotherapy in the management of childhood ACT has not 
been established.11 Similarly, decision in our patient was difficult since the patient exhibit both poor and good prognostic features of an ACT. Taking into consideration the borderline nature of the tumor in the present case and the family's opposition to chemotherapy, we decided to follow-up the patient without adjuvant treatment. Now patient is in complete remission without any complaint at fifth month after surgical resection. Fortunately, with resolution of hyperandrogenism findings and normalization of hormonal profile the patient's mood was improved. Based on the clinical and treatment characteristics of present patient we speculate that, the type of the ACT in our case was borderline in nature. In this type of ACT close follow-up after the total resection of the tumor without chemotherapy and radiotherapy can be a management strategy. However, before coming to strict conclusions, it is necessary to see the long-term results of present case and large patient series including cases similar to our patient and carrying borderline characteristics of ACT.

\section{References}

1. Cordera F, Grant C, van Heerden J, et al. Androgen-secreting adrenal tumors. Surgery 2003;134:874-80.

2. Sutter JA, Grimberg A. Adrenocortical tumors and hyperplasias in childhood-etiology, genetics, clinical presentation and therapy. Pediatr Endocrinol Rev 2006;4:329.

3. Wooten MD, King DK. Adrenal cortical carcinoma: Epidemiology and treatment with mitotane and a review of the literature. Cancer 1993;72:3145-55.

4. Ciftci A0, Senocak ME, Tanyel FC, Büyükpamukçu N. Adrenocortical tumors in children. J Pediatr Surg 2001; 36:549-54.

5. Michalkiewicz E, Sandrini R, Figueiredo B. et al. Clinical and outcome characteristics of children with adrenocortical tumors: a report from the International Pediatric Adrenocortical Tumor Registry. J Clin Oncol 2004;22:838-45.

6. Weiss LM. Comparative histologic study of
43 metastasizing and nonmetastasizing adrenocortical tumors. Am J Surg Pathol 1984;8:163-9.

7. Medeiros LJ, Weiss LM. New developments in the pathologic diagnosis of adrenal cortical neoplasms. A review. Am J Clin Pathol 1992;97:73-83.

8. Cagle PT, Hough AJ, Pysher TJ, et al. Comparison of adrenal cortical tumors in children and adults. Cancer 1986;57:2235-7.

9. Jain M, Kapoor S, Mishra A, et al. Weiss criteria in large adrenocortical tumors: a validation study. Indian $\mathrm{J}$ Pathol Microbiol 2010;53:222-6.

10. Bugg MF, Ribeiro RC, Roberson PK, et al. Correlation of pathologic features with clinical outcome in pediatric adrenocortical neoplasia. A study of a Brazilian population. Brazilian Group for Treatment of Childhood Adrenocortical Tumors. Am J Clin Pathol 1994; 101:625-9.

11. Ribeiro RC, Michalkiewicz EL, Figueiredo $\mathrm{BC}$, et al. Adrenocortical tumors in children. Braz J Med Biol Res 2000;33:1225-34. 